\title{
Difference in the Foot Intersegmental Coordination Pattern Between Female Lacrosse Players With and Without a History of Medial Tibial Stress Syndrome: a Cross-sectional Study
}

Hiroshi Akuzawa ( $\nabla$ h-akuzawa@asagi.waseda.jp )

Waseda Daigaku https://orcid.org/0000-0002-9316-5570

Tomoki Oshikawa

Waseda Daigaku

Koji Nakamura

Waseda Daigaku

Ren Kubota

Waseda Daigaku

Norifumi Takaki

Waseda Daigaku

Naoto Matsunaga

Seigakuin Daigaku

Koji Kaneoka

Waseda Daigaku

Original Research Article

Keywords: Shin splint, modified vector coding technique, sports, biomechanics, risk factor

Posted Date: May 6th, 2020

DOI: https://doi.org/10.21203/rs.3.rs-25912/v1

License: (c) (1) This work is licensed under a Creative Commons Attribution 4.0 International License. Read Full License

Version of Record: A version of this preprint was published at Journal of Foot and Ankle Research on January 31st, 2022. See the published version at https://doi.org/10.1186/s13047-022-00513-y. 


\section{Abstract \\ Background}

Medial tibial stress syndrome is a common sports related injury. Altered foot kinematics can be a risk factor for the injury. Since foot segments can move independently, intersegment coordination is important for proper foot function. This study aimed to compare the foot intersegmental coordination pattern and single segment kinematics between women lacrosse players with and without a history of medial tibial stress syndrome during drop jump.

\section{Methods}

Nine players with a medial tibial stress syndrome history and 12 players with no history were enrolled. Foot kinematics, including angle at landing and peak angle and excursion at the rearfoot, midfoot, and forefoot during single-leg drop jumps were analyzed. Each segment motion data from landing to leaping was time-scaled to $100 \%$ to analyze the intersegmental coordination with a modified vector coding technique. Instant intersegmental coordination of every $1 \%$ was classified into four patterns (in-phase, two segments rotate in the same direction with similar amplitudes; anti-phase, two segments rotate in opposite directions; proximal phase, proximal segment dominantly rotates in the same direction compared to the distal segment; and distal phase, distal segment dominantly rotates in the same direction compared to the proximal segment). The percentage of intersegmental coordination pattern and kinematics in each segment were compared between the groups using the Student's $t$ test.

\section{Results}

Groups with a history of medial stress syndrome showed a significantly higher percentage of proximal phase between the rearfoot and midfoot in the sagittal (Mean \pm SD; history, $52.0 \pm 18.5 \%$, no history, 29.3 $\pm 16.7 \% ; p=0.005$ ) and coronal planes (history, $43.0 \pm 26.1 \%$, no history, $15.9 \pm 9.1 \% ; p=0.008$ ). Dorsiflexion excursion (history, $34.8 \pm 4.7^{\circ}$, no history, $29.6 \pm 2.1^{\circ} ; p=0.002$ ) were significantly larger in a history of medial tibial stress syndrome group compared to no history group.

\section{Conclusions}

Rearfoot dominant motion pattern relative to the midfoot may be related to medial tibial stress syndrome. Intersegmental coordination analysis may be useful for detecting abnormal foot coordination patterns.

\section{Key Points}

Ulntersegmental coordination pattern analysis showed that women athletes with MTSS history had rearfoot dominant motion pattern. 
$\square$ Analysis of single segment kinematics may be insufficient to detect abnormal foot kinematics.

UIntersegmental coordination of the foot should be considered to assess a risk factor of MTSS for future prospective study.

\section{Background}

Medial tibial stress syndrome (MTSS) is one of the most common running-related injuries in athletes. A relatively high incidence rate (13.6-20.0\%) has been reported in athletic populations [1]. A previous study described that the mean recovery time to perform running training program was 105.2-117.6 days [2]. Because of this long recovery time, establishing an effective prevention program for MTSS is required. In order to establish a prevention program, risk factors for MTSS should be clarified. Several risk factors for MTSS have been described in many researches. Navicular drop, which is a midfoot pronation indicator [3], was a common risk factor reported by several systematic reviews $[4,5,6]$. However, navicular drop test only assesses the difference in distance between the floor and the lower border of the unloaded and loaded navicular bones in static postures. This test does not exactly reflect the dynamic motion of the foot during weight bearing condition.

Although assessing dynamic foot motion to predict MTSS risk is important, the foot consists of several segments, such as the rearfoot, midfoot, and forefoot. Each joint individually moves, and the coordination of these intersegmental motions is fundamental to adapt the foot's shape to ground conditions. Because there are complex interactions between the segments during weight bearing activities, there is a limitation to detect abnormal motion pattern by assessing single segment motion. Accordingly, intersegmental coordination pattern analyzes of the foot have been paid attention $[7,8,9]$. The modified vector coding technique can represent coordinated motion patterns between the two adjacent segments [9]. Using this technique, intersegmental coordination pattern between the proximal and distal segments can be classified according to the rotation direction and amplitude. Thus, it can be clarified if two segments rotate in similar or opposite directions, and which segment dominantly rotates relative to the other.

This study aimed to clarify the foot intersegmental coordination pattern and each segmental angle differences between women lacrosse players with and without an MTSS history during drop jump task. It is hypothesized that the players with an MTSS history showed not only different single segment motion pattern, such as excessive eversion, but also altered intersegmental coordination patterns compared with the players without an MTSS history.

\section{Methods}

\section{Participants}

All participants were recruited in a collegiate women lacrosse squad from July 2019 to November 2019. Self-report regarding history of injury was collected from 56 players in the squad by a physical therapist who has a 17-year experience in the field of musculoskeletal and sports physical therapy. MTSS history is 
defined by the following criteria: 1) players experienced an atraumatic occurrence of pain and tenderness in the distal two-thirds of the medial tibia, which lasted at least 1 week; 2 ) pain was aggravated by running; and 3) training was limited by pain.[10] No history of lower limb injuries was defined as absence of any pain or trauma in the lower limbs, which may disturb lacrosse training. If players had ongoing symptoms, which met the criteria during research period, these players were excluded. Nine players had an MTSS history. Among them, three players had an MTSS history in both legs. In those cases, the two legs were separately counted as independent leg. Also, twelve players who had no history of lower limb injuries were recruited. Thus, data from 12 legs with MTSS history (MTSS history group) and 12 legs without any history of injuries (no history group) were collected in this study (Table 1). All the participants provided written informed consent prior to participating.

\section{Three Dimensional Assessment}

Three dimensional assessments were performed at the University's laboratory. Twelve and four reflective markers were attached to the anatomical landmarks of the foot and shank, respectively, according to the Rizzoli Foot Model (RFM) [11]. Eight infrared cameras (Oqus; QUALIYSIS, Göteborg, Sweden) were set to collect marker trajectories on the foot and shank. Double-leg standing posture for $5 \mathrm{~s}$ was recorded to normalize the angles during the drop jump. Then, the players were instructed to perform a single-leg drop jump from a 30-cm box. Sufficient practice was allowed for the players to be familiar with the task before the measurement. Verbal instruction was provided to the players that they drop off the box and leap up vertically as high as possible immediately after landing. All the participants showed forefoot contact during drop jump. A force plate (Kistler, Winterthur, Switzerland) was set in front of the box to decide the landing and take-off timings. Three successful drop jumps were recorded at a sampling rate of $200 \mathrm{~Hz}$.

\section{Data Processing}

The segment angle data from landing to leaping, which were decided based on the ground reaction force data measured by the force plate, were selected and analyzed. The ground reaction force data were filtered with a low-pass filter at $12 \mathrm{~Hz}$. A vertical component of the ground reaction force $>10 \mathrm{~N}$ was defined as the point of landing on the force plate [12]. The point at which the force became $<10 \mathrm{~N}$ after the landing was defined as the point of leaping. The segment angle data were analyzed using the Visual 3D software (C-motion Inc., Maryland, USA). Three-dimensional (3D) marker trajectories were filtered using a fourth-order Butterworth low-pass filter with a 6-Hz cut-off frequency [13]. The shank, rearfoot, midfoot, and forefoot segments were created according to the RFM [11]. Orientations of X-axis pointing forward, $Y$-axis pointing upward, and Z-axis pointing to the right according to a standardization proposal [14] (Fig. 1). A Cardan sequence (Z-X-Y, representing dorsi-plantar flexion, eversion-inversion, and abduction-adduction) was used to calculate relative angles between segments. A positive value represents dorsiflexion, eversion, and abduction and a negative value means plantar flexion, inversion, and adduction. Three-dimensional rearfoot segment rotation relative to the shank was defined as rearfoot motion. Three-dimensional midfoot segment rotation relative to the rearfoot was defined as midfoot motion. Moreover, three-dimensional forefoot segment rotation relative to the midfoot was defined as 
forefoot motion. The segment angles of the rearfoot, midfoot, and forefoot were calculated. Since the segment motion in the transverse plane was relatively small, only the sagittal and coronal plane motions were calculated. The segment angles were normalized to the static double-leg standing posture. The segment angles at landing and peak angles from landing to leaping were calculated. Segment excursion was measured as the difference between the angle at the landing and peak angle in the sagittal and coronal planes. Also, the segment angle data from landing to leaping in each trial were time-scaled to $100 \%$ for intersegmental coordination analysis.

\section{Repeatability Assessment}

Four players (one in the MTSS history group and three in the no history group) participated in the assessment for measurement repeatability. Repeated measurement was conducted more than a month after the first measurement. In order to assess the similarity of averaged kinematic waveforms of the foot segments acquired by two measurements, the coefficient of multiple correlation (CMC) was employed $[15,16]$. The kinematic data from 5 feet from 4 participants were employed for repeatability analysis. The $\mathrm{CMC}$ value of 1 means perfect match and undefined value indicates dissimilar waveforms [16].

\section{Intersegmental Coordination Analysis}

To calculate intersegmental coordination, the modified vector coding technique was utilized [8,9]. A coupling angle $(Y i)$ for each instant (i) during the normalized drop jump task was calculated by this technique to quantify intersegmental coordination according to Equations (1) and (2) [13].

$$
\begin{aligned}
& \gamma_{i}=\operatorname{Atan}\left(\frac{\theta_{D(i+1)}-\theta_{D i}}{\theta_{P(i+1)}-\theta_{p i}}\right) \frac{180}{\pi} \text { if } \theta_{P(i+1)}-\theta_{p i}>0 \\
& \gamma_{i}=\operatorname{Atan}\left(\frac{\theta_{D(i+1)}-\theta_{D i}}{\theta_{P(i+1)}-\theta_{p i}}\right) \frac{180}{\pi}+180 \text { if } \theta_{P(i+1)}-\theta_{p i}<0
\end{aligned}
$$

Coupling angle $(Y i)$ was calculated to show a value of $0-360^{\circ}$ according to Equation (3).

$$
\gamma_{i}= \begin{cases}\gamma_{i}+360 & \text { if } \gamma_{i}<0 \\ \gamma_{i} & \text { if } \gamma_{i} \geqq 0\end{cases}
$$

The mean coupling angle $(Y i)$ of three trials was calculated using circular statistics of Equations (4) and (5).

$$
\begin{aligned}
& \bar{x}_{i}=\frac{1}{n} \sum_{i=1}^{n} \cos \gamma_{i} \\
& \bar{y}_{i}=\frac{1}{n} \sum_{i=1}^{n} \sin \gamma_{i}
\end{aligned}
$$

The following conditions (6) were applied to calculate the mean coupling angle ( $Y i)$ of $0-360^{\circ}$. 


$$
\bar{\gamma}_{i}=\left\{\begin{array}{l}
\operatorname{Atan}\left(\frac{y_{i}}{x_{i}}\right) \frac{180}{\pi} \quad \text { if } x_{i}>0, y_{i}>0 \\
\operatorname{Atan}\left(\frac{y_{i}}{x_{i}}\right) \frac{180}{\pi}+180 \text { if } x_{i}<0 \\
\operatorname{Atan}\left(\frac{y_{i}}{x_{i}}\right) \frac{180}{\pi}+360 \text { if } x_{i}>0, y_{i}<0
\end{array}\right.
$$

Instant intersegmental coordination patterns were categorized into one of four patterns according to mean coupling angle $\left({ }^{\overline{\gamma_{i}}}\right)$ : (1) in-phase $\left(22.5 \leqq{ }^{\bar{\gamma}_{i}}<67.5,202.5 \leqq^{\bar{\gamma}_{i}}<247.5\right)$ : the proximal and distal segments rotate in the same direction with similar amplitudes; (2) anti-phase $\left(112.5 \leqq{ }^{\overline{\gamma_{i}}}<157.5,292.5 \leqq\right.$ $\left.\bar{\gamma}_{i}<337.5\right)$, the proximal and distal segments rotate in opposite directions; (3) proximal phase $\left(0 \leqq{ }^{\overline{\gamma_{i}}}<\right.$ $22.5,157.5 \leqq^{\bar{\gamma}_{i}}<202.5,337.5 \leqq^{\overline{\gamma_{i}}} \leqq 360$ ), the proximal segment dominantly rotates compared with the distal segment in the same direction; and (4) distal phase $\left(67.5 \leqq^{\overline{\gamma_{i}}}<122.5,247.5 \leqq^{\bar{\gamma}_{i}}<292.5\right)$, the distal segment dominantly rotates compared with the proximal segment in the same direction [9]. The percentage of each pattern was calculated for each foot.

\section{Sample size calculation}

Statistical power analysis was conducted with the G*power version 3.1 (Heinrich-Heine Universität, Germany). Because there was no previous data which compared intersegmental coordination pattern difference between athletes with and without MTSS history, results from this study were used for a priori power analysis. Minimum sample size was calculated with obtained effect size of 1.3 , which was the minimum value among intersegmental coordination pattern comparison with significant difference, significance level of 0.05 and power level of 0.8 . If sufficient number of participants was not achieved with the measured intersegmental coordination pattern data, further participant recruitment and measurements were planned.

\section{Statistical Analysis}

The SPSS Statistics version 25.0 (IBM, USA) was used for statistical comparison between groups. The Shapiro-Wilk and Levene tests were used to confirm the normal and equal data distributions, respectively. For group comparison in demographic data, angles at landing, peak angles, excursion, and percentage of each intersegmental pattern between the rearfoot and midfoot and the midfoot and forefoot in the sagittal and coronal planes, the Student's t-test, Welch's t-test, or Mann-Whitney U test were employed depending on the normal and equal distributions. The Cohen's $d$ was employed to calculate the effect size for group comparison (effect size: small, 0.2- 0.5; medium, 0.5-0.8; larger, more than 0.8) [17]. The a level was set at 0.05 .

\section{Results}


Statistical power analysis showed that 11 was the minimum sample size for each group. Therefore, further recruitment and measurement was not conducted. There were no significant differences in any demographic data between groups. Repeatability analysis for kinematic waveform showed excellent repeatability for the sagittal plane motions (CMC: rearfoot, 0.97 ; midfoot, 0.98 ; forefoot; 0.91 ) and excellent to good repeatability for the coronal plane motions (CMC: rearfoot, 0.92 ; midfoot, 0.83 ; forefoot; 0.89).

Figure 2 shows the averaged waveform of the rearfoot, midfoot, and forefoot segment angles from landing to leaping during drop jump task in the MTSS history and no history groups. In comparison of percentage of intersegmental coordination patterns between groups (Table 2), percentage of in-phase between the rearfoot and midfoot was significantly lower in the MTSS history group compared with the no history group in the sagittal $(42.2 \pm 17.1 \%$ vs. $65.4 \pm 16.9 \%)$ and coronal planes $(34.0 \pm 15.2 \%$ vs. 58.3 $\pm 17.5 \%$ ). On the contrary, percentage of proximal phase between the rearfoot and midfoot was significantly higher in the MTSS history group compared with the no history group in the sagittal (52.0 \pm $18.5 \%$ vs. $29.3 \pm 16.7 \%$ ) and coronal planes ( $43.0 \pm 26.1 \%$ vs. $15.9 \pm 9.1 \%)$. No significant differences were seen between groups for percentage of anti-phase and distal phase. Furthermore, there were no significant differences between groups for percentage of any phases between the midfoot and forefoot in the sagittal and coronal planes.

Regarding segment angles at landing, peak angles, and excursions, group comparisons are shown in Table 3. The rearfoot plantar flexion angle at landing was significantly greater in the MTSS history group compared with the no history group $\left(-17.6 \pm 3.9^{\circ}\right.$ vs. $\left.-14.6 \pm 2.8^{\circ}\right)$. Moreover, the midfoot plantar flexion angle at landing was significantly smaller in the MTSS group than that in the no history group $\left(-5.7 \pm 3.8^{\circ}\right.$ vs. $\left.-9.2 \pm 3.8^{\circ}\right)$. The MTSS history group showed significantly larger excursion of rearfoot dorsi-plantar flexion $\left(34.8 \pm 4.7^{\circ}\right.$ vs. $\left.29.6 \pm 2.1^{\circ}\right)$ and motions compared with the no history group. In contrast, the MTSS history group demonstrated significantly smaller midfoot excursion of dorsi-plantar flexion (15.4 \pm $3.2^{\circ}$ vs. $\left.9.1 \pm 3.8^{\circ}\right)$ and forefoot eversion/inversion excursion $\left(2.8 \pm 1.0^{\circ}\right.$ vs. $\left.3.9 \pm 0.8^{\circ}\right)$ than that in the no history group.

\section{Discussion}

This is the first study which identified the intersegmental coordination difference between athletes with and without MTSS history. The results of this study showed that the intersegmental coordination percentages between the rearfoot and midfoot were statistically different in the sagittal and coronal plane motions between groups. However, there were no significant differences for segment angle at landing, peak angle, and excursion in eversion/inversion motions. Since the foot consists of many segments, well-coordinated motions between the segments are fundamental to achieve sufficient foot function.

The no history group showed that approximately $60 \%$ of intersegmental coordination patterns in the sagittal and coronal planes between the rearfoot and midfoot were in-phase. This means that the rearfoot 
and midfoot rotated toward same direction with similar amplitude in approximately $60 \%$ of stance phase during drop jump in the no history group [8]. In contrary, segment motions dominantly occurred in the rearfoot compared with the midfoot in the MTSS history group (52\% in sagittal plane and $43 \%$ in coronal plane). Rearfoot motion, especially subtalar joint motion, is important to modulate flexibility and rigidity of the foot, which is so-called "midtarsal joint locking mechanism" [18]. A previous cadaver study demonstrated that mobility of the first, second, and fifth metatarsal joints significantly increased with the calcaneus maximally everted compared with maximally inverted [19]. There is a possibility that more rearfoot rotation might be required relative to the midfoot to obtain proper flexibility and rigidity of the foot for shock absorption and force transmission in the MTSS history group. Since sufficient compensation could be obtained by the rearfoot, intersegmental coordination between the midfoot and forefoot might be similar between groups. Although forefoot excursion in the coronal plane showed significant difference between groups and effect size was large, it should be carefully considered if average difference of $1.1^{\circ}$ has clinical meaning or not.

The single segment angles in the coronal plane, including angle at landing, peak angle, and excursion did not show any difference in the rearfoot and midfoot. This indicates that amount of motion in the rearfoot and midfoot in the coronal plane during drop jump task was similar; however, coordinated motion between the rearfoot and midfoot were different between groups. These results can suggest that assessment of instant angles in single segment may not be able to detect specific kinematic data for athletes with MTSS history. A prospective study detected that the pronation duration during running was the only significant MTSS predictor even though peak eversion angle in the rearfoot was significantly different between the MTSS and control groups [20]. Similar results were seen in this study, such that the MTSS history group showed a higher percentage of rearfoot dominant motion pattern throughout drop jump. Taken together, not only instant single segment kinematic data, such as angles at landing and peak angle, but also coordination pattern throughout the task should be assessed to detect abnormal kinematics in MTSS patients.

Unlike the coronal plane angles, the rearfoot and midfoot angles at landing and excursion in the sagittal plane showed significant difference between groups. These can be interesting findings because coronal plane alignment and motion abnormalities have been mainly reported as risk factor for MTSS by previous studies $[4,5,20]$. A previous study researching each bone kinematics during walking by using bony pins reported similar results that total range of motion in the sagittal plane was $17.0^{\circ}$ at calcaneotibial complex, $8.4^{\circ}$ at navicular-talus joint, and $9.7^{\circ}$ at calcaneocuboid joint [21]. On the other hand, total range of motion in the coronal plane was $11.3^{\circ}$ at calcaneo-tibial complex, $14.9^{\circ}$ at navicular-talus joint, and $11.3^{\circ}$ at calcaneocuboid joint. Because range of motion at the rearfoot in the sagittal plane was larger than that at midfoot, rearfoot might be able to sufficiently compensate less motion at midfoot.

Rearfoot motion is controlled by the calf muscles, including the gastrocnemius, soleus, tibialis posterior, tibialis anterior, flexor digitorum longus, and flexor hallucis longus [22, 23, 24]. One of the possible causes of MTSS is traction force induced longitudinal periostitis produced by the soleus, flexor digitorum longus, and tibialis posterior $[25,26,27]$. It is speculated that excessive motion of the rearfoot leads to higher 
eccentric contraction level of these muscles and produces higher stress on the medial tibia. A previous study showed that running for $30 \mathrm{~min}$ increased the tibialis posterior and flexor digitorum longus stiffness [28]. Also, runners with an MTSS history demonstrated higher tibialis posterior and flexor digitorum longus stiffness than that in no MTSS history [29]. Therefore, rearfoot dominant pattern during drop jump may increase eccentric muscle contraction and traction force to the medial border of the tibia and related to MTSS.

\section{Limitations}

Firstly, this study is a cross-sectional study which cannot identify MTSS risk factor. This study could only present feature of intersegmental coordination pattern of the foot for women lacrosse players with MTSS history. However, the analysis method of this study may be applicable for future prospective study to detect MTSS risk factors. Secondly, since the participants in this study were only women lacrosse players, it is unclear whether same results can be seen in male athletes. Thirdly, because reflective markers were attached on the skin, skin motion artefact can influence the measurement reliability [30]. Even though repeatability of the measurements was good to excellent in this study, it should be carefully understood that these results did not present joint motions.

\section{Conclusions}

This study compared the percentage of intersegmental coordination pattern of the foot and kinematics in each segment during drop jump between women lacrosse players with and without an MTSS history. The MTSS history group showed significantly higher percentage of proximal phase between the rearfoot and midfoot in the sagittal and coronal plane motions. This indicates that the MTSS history group demonstrated rearfoot dominant motion pattern. There is a possibility that rearfoot dominant motion pattern may increase calf muscle contractions to control rearfoot motion, resulting in larger traction force on the medial border of the tibia. Further prospective study is required to detect whether intersegmental coordination difference can be an MTSS risk factor.

\section{Abbreviations}

MTSS: Medial tibial stress syndrome; RFM: Rizzoli Foot Model; CMC: Coefficient of multiple correlation; 95\% Cl: 95\% confidence interval; DF-PF: Dorsiflexion- Plantar flexion, Ev-Inv: Eversion- Inversion.

\section{Declarations}

\section{Ethical approval and consent to participate}

This cross-sectional study was approved by the Ethical Committee in Waseda University (ID: 2019-082). All the participants provided written informed consent prior to participating. This study complied with the Declaration of Helsinki. 


\section{Consent for publication}

Not applicable

\section{Availability of data and material}

The datasets used and/or analysed during the current study are available from the corresponding author on reasonable request.

\section{Competing interests}

The authors, Hiroshi Akuzawa, Tomoki Oshikawa, Koji Nakamura, Ren Kubota, Norifumi Takaki, Naoto Matsunaga, and Koji Kaneoka, declare that they have no competing of interests.

\section{Funding}

This study was supported by KAKENHI (19121623). The funds were used to purchase measurement devices.

\section{Authors' contributions}

$\mathrm{HA}, \mathrm{TO}$, and KK contributed to the study design, data collection, data analysis, interpretation of results and writing manuscript. KN, RK, NT, NM contributed to data collection and data analysis. All authors approved to submit the manuscript and agreed to all aspects of the work.

\section{Acknowledgements}

We would like to thank Editage for English proofreading.

\section{References}

1. Lopes AD, Hespanhol Junior LC, Yeung SS, Costa LO. What are the main running-related musculoskeletal injuries? A Systematic Review. Sports Med. 2012;42(10):891-905.

2. Moen MH, Holtslag L, Bakker E, Barten C, Weir A, Tol JL, et al. The treatment of medial tibial stress syndrome in athletes; a randomized clinical trial. Sports Med Arthrosc Rehabil Ther Technol. 2012;4:12.

3. Reshef N, Guelich DR. Medial tibial stress syndrome. Clin Sports Med. 2012;31(2):273-90.

4. Newman P, Witchalls J, Waddington G, Adams R. Risk factors associated with medial tibial stress syndrome in runners: a systematic review and meta-analysis. Open Access J Sports Med. 2013;4:229-41.

5. Hamstra-Wright KL, Bliven KC, Bay C. Risk factors for medial tibial stress syndrome in physically active individuals such as runners and military personnel: a systematic review and meta-analysis. $\mathrm{Br}$ J Sports Med. 2015;49(6):362-9. 
6. Moen MH, Bongers T, Bakker EW, Zimmermann WO, Weir A, Tol JL, et al. Risk factors and prognostic indicators for medial tibial stress syndrome. Scand J Med Sci Sports. 2012;22(1):34-9.

7. Hafer JF, Boyer KA. Variability of segment coordination using a vector coding technique: Reliability analysis for treadmill walking and running. Gait Posture. 2017;51:222-7.

8. Takabayashi T, Edama M, Yokoyama E, Kanaya C, Kubo M. Quantifying coordination among the rearfoot, midfoot, and forefoot segments during running. Sports Biomech. 2018;17(1):18-32.

9. Chang R, Van Emmerik R, Hamill J. Quantifying rearfoot-forefoot coordination in human walking. J Biomech. 2008;41(14):3101-5.

10. Verrelst R, De Clercq D, Vanrenterghem J, Willems T, Palmans T, Witvrouw E. The role of proximal dynamic joint stability in the development of exertional medial tibial pain: a prospective study. $\mathrm{Br} \mathrm{J}$ Sports Med. 2014;48(5):388-93.

11. Leardini A, Benedetti MG, Berti L, Bettinelli D, Nativo R, Giannini S. Rear-foot, mid-foot and fore-foot motion during the stance phase of gait. Gait Posture. 2007;25(3):453-62.

12. Brund RBK, Rasmussen S, Nielsen RO, Kersting UG, Laessoe U, Voigt M. Medial shoe-ground pressure and specific running injuries: A 1-year prospective cohort study. J Sci Med Sport. 2017;20(9):830-4.

13. Needham R, Naemi R, Chockalingam N. Quantifying lumbar-pelvis coordination during gait using a modified vector coding technique. J Biomech. 2014;47(5):1020-6.

14. Wu G, Cavanagh PR. ISB recommendations for standardization in the reporting of kinematic data. J Biomech. 1995;28(10):1257-61.

15. Alberto Ferrari AGC, Angelo Cappello. A new formulation of the coefficient of multiple correlation to assess the similarity of waveforms measured synchronously by different motion analysis protocols. Gait Posture. 2010;31:540-2.

16. Kadaba MP, Ramakrishnan HK, Wootten ME, Gainey J, Gorton G, Cochran GV. Repeatability of kinematic, kinetic, and electromyographic data in normal adult gait. J Orthop Res. 1989;7(6):849-60.

17. Cohen J. A power primer. Psychol Bull. 1992;112(1):155-9.

18. Elftman H. The transverse tarsal joint and its control. Clin Orthop. 1960;16:41-6.

19. Blackwood CB, Yuen TJ, Sangeorzan BJ, Ledoux WR. The midtarsal joint locking mechanism. Foot Ankle Int. 2005;26(12):1074-80.

20. Becker J, Nakajima M, Wu WFW. Factors Contributing to Medial Tibial Stress Syndrome in Runners: A Prospective Study. Med Sci Sports Exerc. 2018;50(10):2092-100.

21. Lundgren P, Nester C, Liu A, Arndt A, Jones R, Stacoff A, et al. Invasive in vivo measurement of rear-, mid- and forefoot motion during walking. Gait Posture. 2008;28(1):93-100.

22. Klein P, Mattys $S$, Rooze M. Moment arm length variations of selected muscles acting on talocrural and subtalar joints during movement: an in vitro study. J Biomech. 1996;29(1):21-30.

23. Sammarco VJ. The talonavicular and calcaneocuboid joints: anatomy, biomechanics, and clinical management of the transverse tarsal joint. Foot Ankle Clin. 2004;9(1):127-45. 
24. Hintermann B, Nigg BM, Sommer C. Foot movement and tendon excursion: an in vitro study. Foot Ankle Int. 1994;15(7):386-95.

25. Franklyn M, Oakes B. Aetiology and mechanisms of injury in medial tibial stress syndrome: Current and future developments. World J Orthop. 2015;6(8):577-89.

26. Moen MH, Tol JL, Weir A, Steunebrink M, De Winter TC. Medial Tibial Stress Syndrome a critical review. Sports Med. 2009;39(7):523-46.

27. Bouche RT, Johnson $\mathrm{CH}$. Medial tibial stress syndrome (tibial fasciitis): a proposed pathomechanical model involving fascial traction. J Am Podiatr Med Assoc. 2007;97(1):31-6.

28. Ohya S, Nakamura M, Aoki T, Suzuki D, Kikumoto T, Nakamura E, et al. The effect of a running task on muscle shear elastic modulus of posterior lower leg. J Foot Ankle Res. 2017;10:56.

29. Saeki J, Nakamura M, Nakao S, Fujita K, Yanase K, Ichihashi N. Muscle stiffness of posterior lower leg in runners with a history of medial tibial stress syndrome. Scand J Med Sci Sports. 2017;28(1):246-51.

30. Novak AC, Mayich DJ, Perry SD, Daniels TR, Brodsky JW. Gait analysis for foot and ankle surgeonstopical review, part 2: approaches to multisegment modeling of the foot. Foot Ankle Int. 2014;35(2):178-91.

\section{Tables}

Table 1. Participant characteristics

\begin{tabular}{|c|c|c|}
\hline & MTSS history & No history \\
\hline Age, $y^{a}$ & $20 \pm 1$ & $20.9 \pm 1$ \\
\hline Height, $\mathrm{cm}^{\mathrm{a}}$ & $159.8 \pm 2.5$ & $160.5 \pm 5.2$ \\
\hline Weight, $\mathrm{kg}^{\mathrm{a}}$ & $53.1 \pm 3.6$ & $55.7 \pm 6.3$ \\
\hline Measured Foot ${ }^{b}$ (Right/ Left) & $8 / 4$ & $8 / 4$ \\
\hline
\end{tabular}

${ }^{a}$ Values are mean $\pm \mathrm{SD},{ }^{\mathrm{b}}$ Values are $\mathrm{n}$. 
Table 2 Percentage of inter-segment coordination pattern comparisons between gro

\begin{tabular}{|c|c|c|c|c|c|c|c|}
\hline Patterns & $\begin{array}{c}\text { Inter- } \\
\text { segments }\end{array}$ & Motions & $\begin{array}{l}\text { MTSS } \\
\text { history }^{\text {a }}\end{array}$ & $\begin{array}{c}\text { No } \\
\text { history }^{\mathrm{a}}\end{array}$ & $p$ value & 95 & $\begin{array}{l}\text { Effect } \\
\text { size }\end{array}$ \\
\hline \multirow{4}{*}{ In-phase } & Rear- mid & DF-PF & $42.2 \pm 17.1$ & $65.4 \pm 16.9$ & $* 0.003$ & -37.6 to -8.5 & 1.4 \\
\hline & & Ev-Inv & $34.0 \pm 15.2$ & $58.3 \pm 17.5$ & $* 0.001$ & 38.1 to -10 & 1.5 \\
\hline & Mid-fore & DF-PF & $24.9 \pm 20.6$ & $22.8 \pm 18.5$ & 0.399 & -14.5 to $18 \therefore$ & 0.1 \\
\hline & & Ev-Inv & $12.3 \pm 7.3$ & $8.3 \pm 5.5$ & 0.207 & -1.5 to 9.5 & 0.6 \\
\hline \multirow{4}{*}{$\begin{array}{l}\text { Anti- } \\
\text { phase }\end{array}$} & Rear- mid & DF-PF & $3.0 \pm 2.1$ & $3.1 \pm 1.5$ & 0.913 & -1.7 to 1.5 & 0.1 \\
\hline & & Ev-Inv & $9.8 \pm 9.0$ & $9.8 \pm 6.6$ & 0.621 & -6.8 to 6.6 & 0 \\
\hline & Mid- fore & DF-PF & $12.0 \pm 10.6$ & $8.9 \pm 6.4$ & 0.399 & -4.4 to 10.6 & 0.4 \\
\hline & & Ev-Inv & $41.6 \pm 19.3$ & $50.9 \pm 15.8$ & 0.207 & -24.2 to 5.6 & 0.5 \\
\hline \multirow{4}{*}{$\begin{array}{l}\text { Proximal } \\
\text { phase }\end{array}$} & Rear- mid & DF-PF & $52.0 \pm 18.5$ & $29.3 \pm 16.7$ & $* 0.005$ & 7.7 to 37.6 & 1.3 \\
\hline & & Ev-Inv & $43.0 \pm 26.1$ & $15.9 \pm 9.1$ & $* 0.008$ & 9.9 to 44.3 & 1.4 \\
\hline & Mid- fore & DF-PF & $59.3 \pm 18.1$ & $63.1 \pm 17.9$ & 0.615 & -19.0 to $11 .$. & 0.2 \\
\hline & & Ev-Inv & $30.1 \pm 25.1$ & $29.3 \pm 15.0$ & 0.665 & -16.7 to 18.2 & 0 \\
\hline \multirow{4}{*}{$\begin{array}{l}\text { Distal } \\
\text { phase }\end{array}$} & Rear- mid & DF-PF & $2.8 \pm 2.3$ & $2.1 \pm 1.6$ & 0.498 & -1.0 to 2.3 & 0.3 \\
\hline & & Ev-Inv & $13.2 \pm 18.6$ & $15.8 \pm 8.8$ & 0.068 & -15.0 to 9.6 & 0.2 \\
\hline & Mid- fore & DF-PF & $3.7 \pm 3.7$ & $5.1 \pm 4.8$ & 0.336 & -5.0 to 2.2 & 0.3 \\
\hline & & Ev-Inv & $15.9 \pm 12.8$ & $11.4 \pm 5.6$ & 0.525 & -4.1 to 13.1 & 0.5 \\
\hline
\end{tabular}

Abbrebiation: DF-PF, Dorsiflexion- plantar flexion, Ev-Inv, Eversion- Inversion,

${ }^{\mathrm{a}}$ Valuses are mean $\pm \mathrm{SD}$, *Significant differences between groups.

Table 3 Segment angle (in degree) comparisons between groups

\begin{tabular}{|c|c|c|c|c|c|c|c|}
\hline & Segments & Motions & $\begin{array}{c}\text { MTSS } \\
\text { history }^{\text {a }}\end{array}$ & $\begin{array}{c}\text { No } \\
\text { history }^{\mathrm{a}}\end{array}$ & $\mathrm{p}$ value & $95 \% \mathrm{CI}$ & $\begin{array}{c}\text { Effect } \\
\text { size }\end{array}$ \\
\hline \multirow{6}{*}{$\begin{array}{l}\text { Angle at } \\
\text { landing }\end{array}$} & \multirow[t]{2}{*}{ Rearfoot } & DF-PF & $-17.6 \pm 3.9$ & $-14.6 \pm 2.8$ & $* 0.039$ & -5.9 to -0.2 & 0.9 \\
\hline & & Ev-Inv & $-3.0 \pm 2.8$ & $-2.3 \pm 3.1$ & 0.617 & -3.1 to 1.9 & 0.2 \\
\hline & \multirow[t]{2}{*}{ Midfoot } & DF-PF & $-5.7 \pm 3.8$ & $-9.2 \pm 3.8$ & $* 0.016$ & 0.7 to 6.4 & 1.1 \\
\hline & & Ev-Inv & $-0.01 \pm 2.1$ & $-0.7 \pm 3.4$ & 0.578 & -1.8 to 3.1 & 0.2 \\
\hline & \multirow[t]{2}{*}{ Forefoot } & DF-PF & $-3.3 \pm 1.8$ & $-3.0 \pm 2.3$ & 0.720 & -2.1 to 1.5 & 0.2 \\
\hline & & Ev-Inv & $2.2 \pm 1.4$ & $3.1 \pm 1.5$ & 0.113 & -2.1 to 0.2 & 0.7 \\
\hline \multirow{6}{*}{$\begin{array}{l}\text { Peak } \\
\text { angle }\end{array}$} & \multirow[t]{2}{*}{ Rearfoot } & DF-PF & $17.2 \pm 3.0$ & $15.0 \pm 2.7$ & 0.084 & -0.3 to 4.6 & 0.7 \\
\hline & & Ev-Inv & $5.6 \pm 2.4$ & $3.9 \pm 4.1$ & 0.216 & -1.1 to 4.5 & 0.5 \\
\hline & \multirow[t]{2}{*}{ Midfoot } & DF-PF & $9.7 \pm 2.5$ & $9.9 \pm 2.6$ & 0.847 & -2.4 to 1.9 & 0.1 \\
\hline & & Ev-Inv & $5.1 \pm 1.7$ & $5.4 \pm 3.1$ & 0.770 & -2.4 to 1.8 & 0.1 \\
\hline & \multirow[t]{2}{*}{ Forefoot } & DF-PF & $2.4 \pm 1.3$ & $2.9 \pm 1.1$ & 0.354 & -1.5 to 0.6 & 0.4 \\
\hline & & Ev-Inv & $-0.6 \pm 1.2$ & $-0.8 \pm 1.2$ & 0.702 & -0.8 to 1.2 & 0.2 \\
\hline \multirow[t]{6}{*}{ Excursion } & \multirow[t]{2}{*}{ Rearfoot } & DF-PF & $34.8 \pm 4.7$ & $29.6 \pm 2.1$ & $* 0.002$ & 2.1 to 8.3 & 1.4 \\
\hline & & Ev-Inv & $8.6 \pm 3.6$ & $6.2 \pm 2.1$ & 0.067 & -0.2 to 4.9 & 0.8 \\
\hline & \multirow[t]{2}{*}{ Midfoot } & DF-PF & $15.4 \pm 3.2$ & $19.1 \pm 3.8$ & $* 0.016$ & -6.7 to 0.8 & 1.1 \\
\hline & & Ev-Inv & $5.2 \pm 2.5$ & $6.1 \pm 1.6$ & 0.276 & -2.8 to 0.8 & 0.5 \\
\hline & \multirow[t]{2}{*}{ Forefoot } & DF-PF & $5.8 \pm 2.0$ & $5.9 \pm 2.1$ & 0.858 & -1.9 to 1.5 & 0.1 \\
\hline & & Ev-Inv & $2.8 \pm 1.0$ & $3.9 \pm 0.8$ & $* 0.006$ & -0.4 to -1.9 & 1.2 \\
\hline
\end{tabular}

Abbrebiation: DF-PF, Dorsiflexion- plantar flexion, Ev-Inv, Eversion- Inversion,

${ }^{a}$ Valuses are mean $\pm \mathrm{SD}$, *Significant differences between groups. 


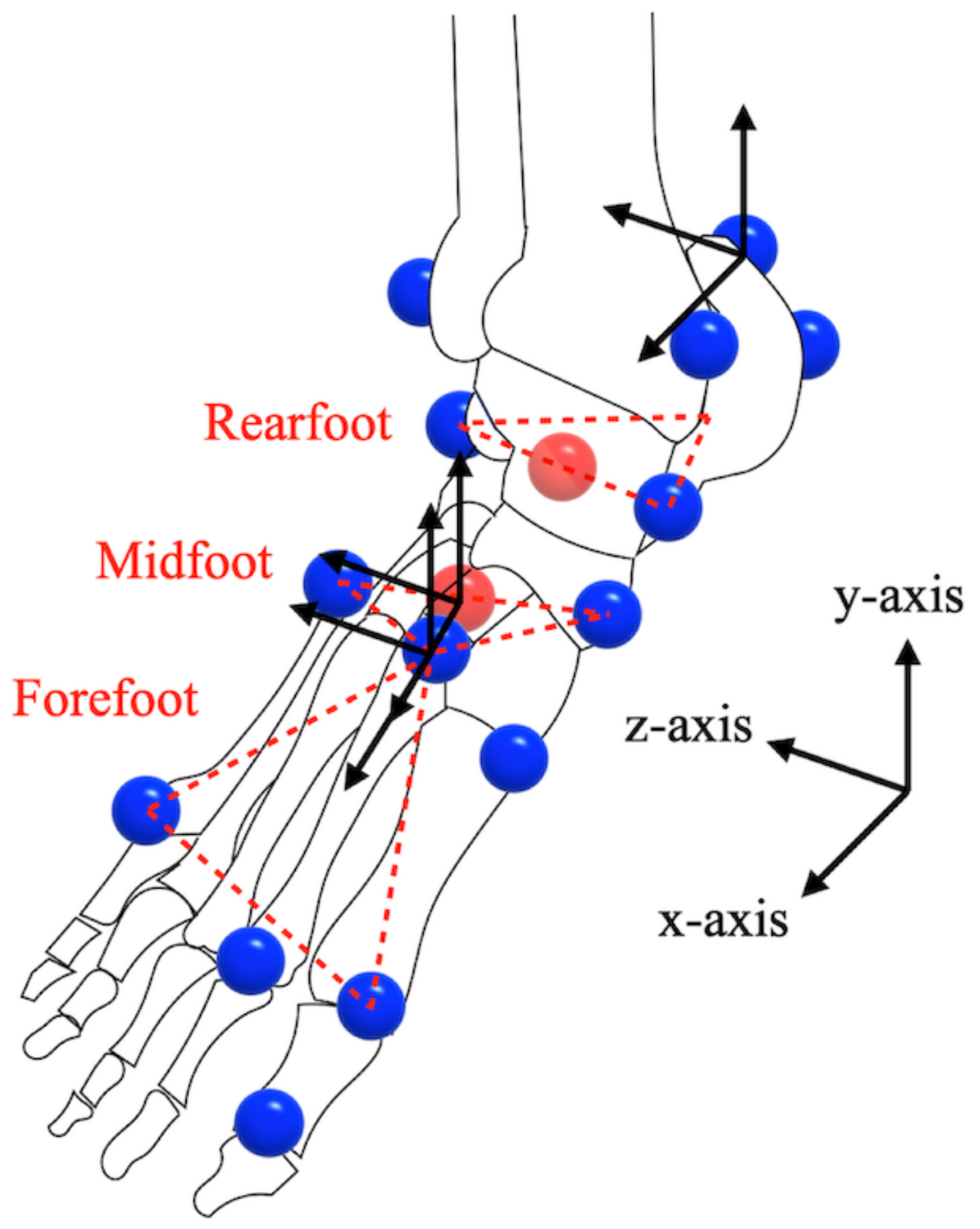

\section{Figure 1}

Marker setting and orientation of each axis in each foot segment. Red dashed lines show each segment (the rearfoot, midfoot, and forefoot). Blue spheres represent reflective markers attached on the landmarks. Red spheres indicate the mid points between the sustentaculum tali of calcaneus and lateral apex of the peroneal tubercle, and medial apex of the tuberosity navicular and head of 5 th metatarsus. 

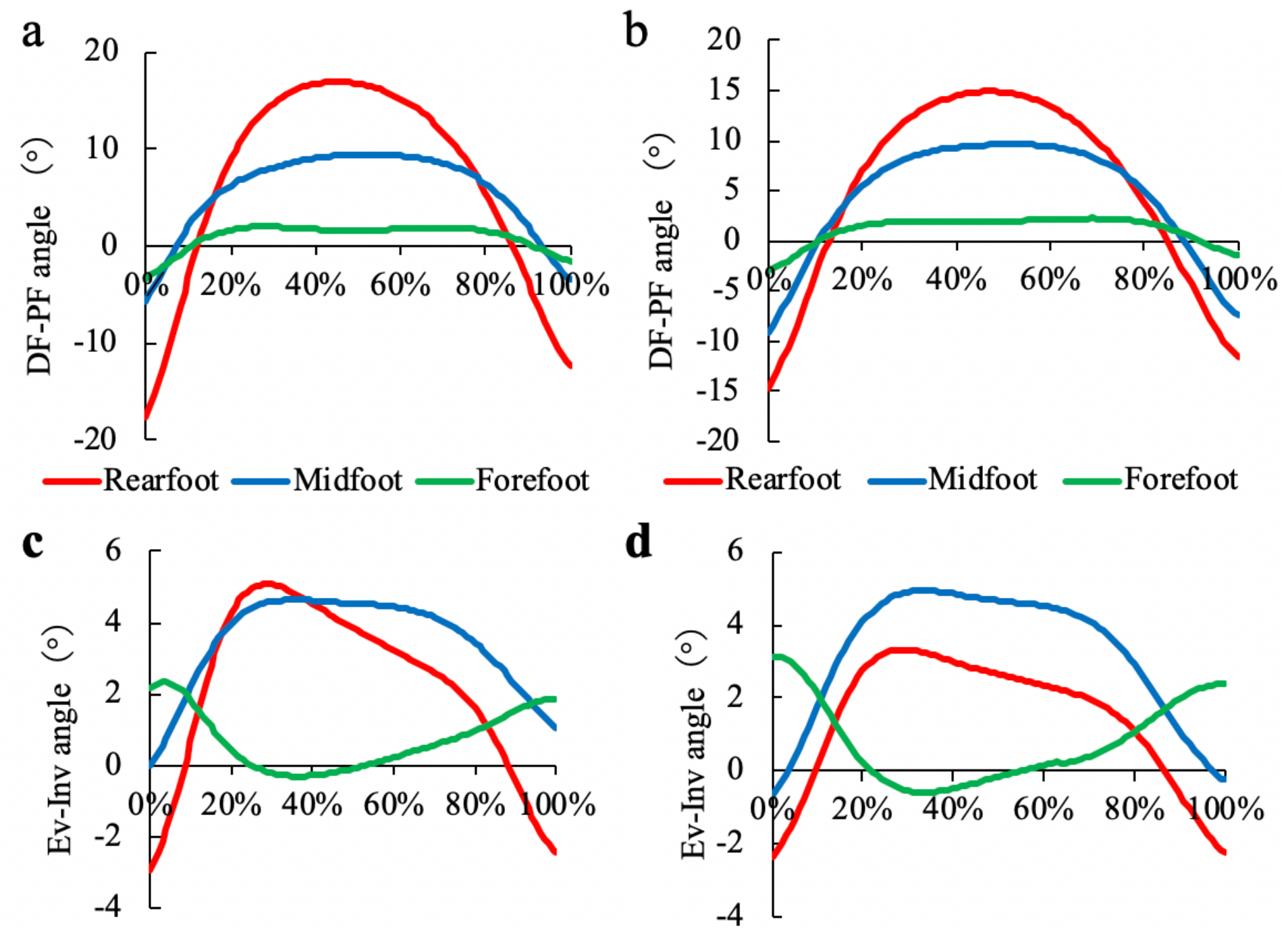

- Rearfoot $\longrightarrow$ Midfoot $\longrightarrow$ Forefoot

-Rearfoot —Midfoot $\longrightarrow$ Forefoot

Figure 2

Averaged waveform of the rearfoot, midfoot, and forefoot segment angles in the MTSS history and no history groups. a: Dorsi-plantar flexion angle in the MTSS history group, b: Dorsi-plantar flexion angle in the no history group, c: Eversion- Inversion angle in the MTSS group history, d: Eversion-Inversion angle in the no history group. Positive value: dorsiflexion and eversion. Negative value: plantar flexion and inversion. 0\%: Landing, 100\%: Taking-off. 